\title{
A Study and Critical Survey on Service Reusability Metrics
}

\author{
T. Karthikeyan \\ P.S.G. College of Arts and Science, Coimbatore, India \\ Email: t.karthikeyan.gasc@gmail.com \\ J. Geetha \\ Sri Manakula Vinayagar Engineering College, Puducherry, India \\ Email: geethakishorekumar@gmail.com
}

\begin{abstract}
The software productivity and quality can be improved by the systematic reuse of software. Reuse and reusability are the two important factors in the software development. Reusability is a prime principle in service oriented architecture. For realizing the reuse of service, the adequacy of ability of service reuse should be properly quantified. The quantification of reuse needs a suite of metrics and many metrics are also available. Each research and publication focuses on different qualities of service to propose a set of metrics for service reusability. This paper studies the service reusability to understand the concept and survey the reusability metrics. The purpose of the paper is exploring the available service reusability metrics for further research.
\end{abstract}

Index Terms-Service Reusability, Reusability metrics, Study and survey of reusability

\section{Introduction}

Software reuse is the using the previously developed software for building of newly developing system [1]. Without reuse many basic business problems cannot be achieved in time. The concept of reuse is not a new one. Many manufacturing industries have been adopted reuse of already developed component to improve quality and to reduce development cost and time. In software industry, the evolution of reusability started from Object Oriented Systems, then Component based Systems and now it talks with Service Oriented Systems. The increasing demand of development of new software applications leads to software reuse. The reuse may be opportunistic reuse or systematic reuse [2]. In opportunistic reuse the developer has to take the decision to find the reusable element, retrieve and reuse it. Whereas systematic reuse is a planned reuse in which the specific component is identified as a reusable one at specific location. To make use of the software as reusable one, we must consider all possible scenarios in the development of software product. The program should be more generic. The program must be a multipurpose program. The rest of the paper is organized as follows. Section 2 studies the service reusability. Section 3 describes the characteristics of service reusability. In section 4 we survey the service reusability metrics. Section 5 concludes the paper.

\section{Service Reusability}

Reusability is the most demanding core principle of Service Oriented Architecture (SOA). Service reusability is the measure of ease with which one can use previously developed services in the new applications. The service can be reused in different tasks. It may be reused in the same system at the different level or it may be reused in many other systems. The development of service is not for a single consumer but for many other consumers [3]. The service designer should focus on the requirements of more consumers rather than the initial service consumer requirements [4]. Therefore the emphasis is given on service reusability. Service reusability is the main goal in SOA. Designing service to be reusable will increase Return On Investment (ROI) and also it reduces the cost associated with design, development, software testing and maintenance [3].The definition of service reusability can be given by many authors.Erl [3] defined as, "Services contain and express agnostic logic and can be positioned as reusable enterprise resources.”In [5], the author defined as, "Reusability of service is the degree to which the service can be used in more than one business 
process or service applications without having much overhead to discover configure and invoke it.”

\subsection{Service Design Attributes for Reusability}

1. The design should be more as generic as possible and it should cover current and future requirements of the consumer. Different application uses should be considered in a service design [6].

2. The Service should be designed for unknown requirements.

3. The service interface contract should be more generalized and standard compliant, so that it can communicate with many consumers.

4. Service should be easily understandable. More understandable service can be captured by many consumers.

5. Functionally the service should be rich.

6. It should possess good exception handling.

7. Service should easily be portable.

8. The reusable service should be independent of other services and business process.

\subsection{Benefits of Service Reuse}

1. Reuse reduces the development time and cost.

2. Reuse minimizes the duplication of services.

3. Reusing the service makes maintenance easier.

4. Reusing of service increases the productivity thereby increase Return On Investment.

5. The reliability of the service increases and overall risk reduces.

6. By reusing, a new business process can be assembled from the existing services to meet the current consumer's requirements and market needs.

\subsection{Misconceptions}

1. The size and complexity of the reusable service is more.

2. Reusable service is always coarse grained.
3. Reusable service may contain much functionality than others.

4. If service is not reusable, it is not properly designed.

5. If service is reusable, the performance will be decreased.

6. If service is reusable, the maintenance will be increased.

7. Identification of reusable service is difficult.

\section{Characteristics of Service Reusability}

For making the service as a reusable one, the emphasis should be given to both design and quality characteristics (Fig.-1). At the design stage the service should be adhere oth $\cdot r$ design characteristics which enhance service reusability.

\subsection{Design Characteristics of Service Reusability}

- Loose Coupling: Service loose coupling enhances the service reusability. The lower the dependency with other services, the more easily it can be reused.

- Composability: Service composability is the key principle for reusability. The composable service can easily aggregate with other services. Therefore the service composability offers higher degree of service reusability.

- Autonomy: The reusable service should be autonomous. If the service is independent from other services and business logic and self governance, then the service will be more reusable.

- Abstraction: Service abstraction hides the unnecessary information from the service consumers. Also it reduces the needless coupling between the service consumer and service provider thereby increases the service reusability.

- Statelessness: Statelessness encourages service reusability. Lesser the amount of state management responsibilities increases its 
scalability and availability which are the required qualities to enhance service reusability.

- Discoverability: Service discoverability promotes service reusability. If and only if the service consumer can easily find the required service, the service can be more reusable.

- Granularity: The service granularity may be fine grained or coarse grained. Depending upon the type of service, the granularity level may vary. The correct granularity level of the service enhances the service re-use.

\subsection{Quality characteristics of Service Reusability}

In software quality standard ISO/IEC 9126, Reusability quality is not included. As far as the service oriented architecture is concerned, the reusability is an important quality. Therefore we should include reusability in the quality model. By keeping the attributes of reusability in mind, we can include quality characteristics of reusability as,

- Generality: The service should be more generic.

The reusable service is not only developed for current requirements and also for unknown future requirements.

- Understandability: The service and its interface should be easily understandable by the user [7]. More understandable, more consumers will be there.

- Functionality: The service should be functionally rich. To have a highest degree of guaranteed reuse, the service should pack with a complete range of functionality.

- $\quad$ Reliability: Since the service is used by many consumers, it should be reliable. The service should able to provide the continuous service to the consumer without any failure.

- Portability: The portable services which are to be consumed through any kind of environment without being modified increases service reusability.
- Availability: The service should be readily available to the consumer for utilization of service.

The easily available service can only be reusable.

From the above discussion it is reveal that the above discussed design and quality characteristics are determinants of the service reusability. Therefore the evaluation of service reusability should consider the above characteristics.

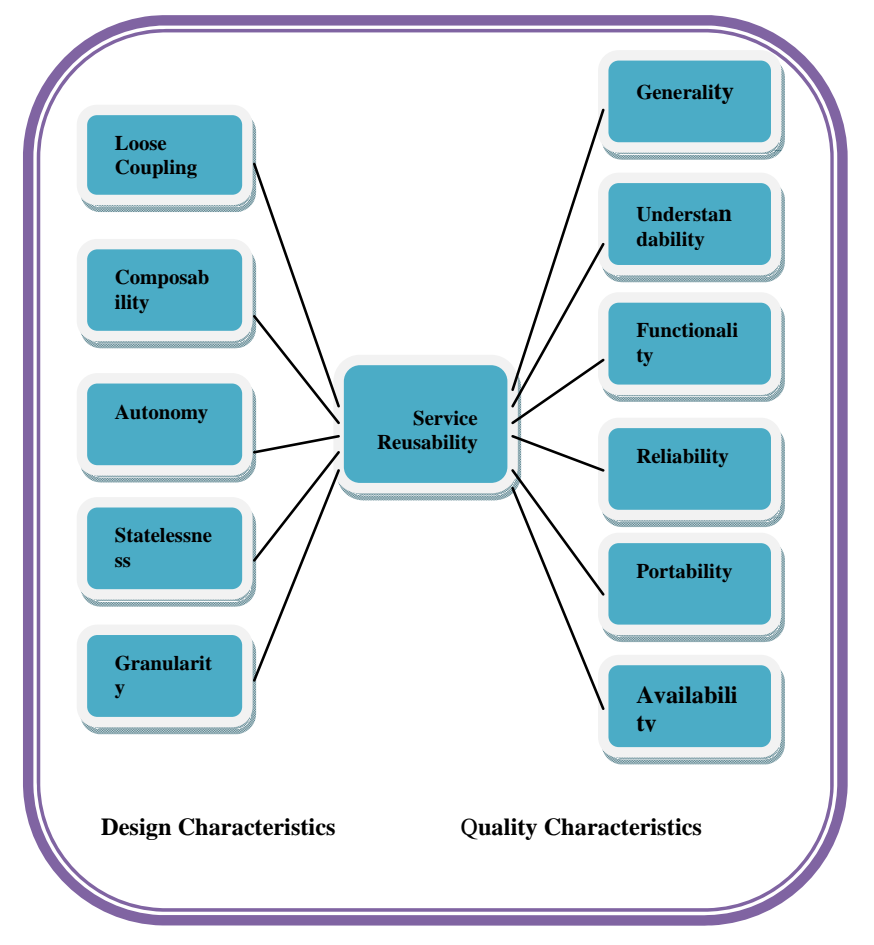

Figure - 1 Service Reusability Characteristics

\section{Reusability Metrics}

Software metric is used for measuring the software's quality and performance quantitatively. To realize the service reuse, we are in need of measuring service reusability. Software metrics are used to measure reusability quantitatively. Many metrics and methods have already been proposed by many authors. In [8] the authors describes that the standardization of functionality increases the reuse of services. Arun Sharma et al [9] say that indirectly the reusability can be measured through complexity, adaptability and observability.

- Khoshkbarforoushha et al [4] a metric for composite service reusability based on logic and description 
mismatch. The paper describes that a given composite service cannot be reused if there is mismatch between consumers' requirement and service providers. The potential reusability metric is given as in eqn. (1),

$$
R_{p}=R_{c} \times\left[1-M M P_{s}\right]
$$

The potential reusability can be measured in terms of number of process reused in the current context $\left(R_{\mathrm{c}}\right)$ and mismatch probability $\left(M M P_{\mathrm{s}}\right)$. This mismatch probability is measured by mismatch probability of service logic and service description.

$>$ Service description mismatch is mismatch between the consumer's requirements and description of the given service.

> Service Logic mismatch is mismatch between the consumer's requirements and logic of the given service.

The paper also validates the metric using experiment model using two separate projects. This paper limits with the probability of availability of service.

- Bingu Shim et al [10] propose a design quality model and metrics for assessing the quality attributes of service oriented architecture. The authors proposed metrics for quality attributes like effectiveness, understandability, flexibility and reusability. The metrics were derived from quality metrics used in QMOOD. For deriving metrics for SOA, the design properties are identified which are derived from SOA design Componentsservices, operations, messages, and connection among services. From the SOA design properties they derived metrics for high level design quality attributes. The metric for reusability is derived as in (2).

Reusability $=-0.5 *$ Coupling $+0.61 *$ Service Granularity + $0.61 *$ Parameter Granularity

The design properties Coupling, Service Granularity and Parameter Granularity are measured in terms of Number of Messages Used, Number of Synchronous Operations, Number of Asynchronous Operations, Number of Directly Connected Producer
Services, Number of Directly Connected Consumer Services, System Size in Number of Services and Number of Fine Grained Parameter Operations.

This paper also conducted an empirical evaluation over a research information management system (RIMS) in service for a research agency. The metrics were evaluated and compared over the different versions of the same system. This reusability metric takes only few design properties like coupling, service granularity and parameter granularity and remaining properties are not considered.

- R. Sindhgatta et al [11] proposed reusability metric based on use of the service by service consumers. This paper considers at design stage, the services which are coupled with the service as consumers. They have given the reusability metric as the number of consumers coupled with the service is mentioned as service reuse index. The metric Service Reuse Index is given as in (3),

$$
\operatorname{SRI}(s)=\left|\mathrm{S}_{\text {consumers }}(s)\right|
$$

This paper concludes with empirical study to measure the proposed metrics. The metric considers only the coupling between services at the design stage.

- Huynh Quyet Thang et al [12] developed a suite of reuse metrics based on metrics proposed by Washizaki for measuring component reuse [13]. The authors include the quality factors such as Understandability, Adaptability, Portability and also Flexibility. Based on these the authors were proposed a suite of metrics for measuring reusability and they are :

o Exist of Meta Information (EMI): This metric can be used by the consumer to understand the service usage.

o Rate of Service Observability (RSO): This metric to measure how ease to observe the service by means of its operations and its input and output parameters. 
o Rate of Service Customizability (RSC): It measures the degree of service's customization.

o Self - Completed of Service's return Value (SCSr): This metric measures the return value of service which is the objective of reuse.

o Self - Completed of Service's parameter (SCSp): This measures self completeness of service and measured by means of the percentage of business methods without any parameter in all business methods which are implemented in the measured service.

o Density of Multi-Grained Method (DMG): This metric measures the percentage of the sum of overloaded functions in the sum of different tasks.

All the metric values range from 0 and 1 . Higher the value, the measured service will have high reusability.

- Si Won Choi and Soo Dong Kim [14] proposes a quality model for evaluating reusability of services by introducing metrics. They have given the reusability (RE) metric as in (4),

$$
\begin{aligned}
\mathrm{RE}= & \mathrm{BCM} \times\left(M D \times W_{\mathrm{MD}}+A D \times W_{\mathrm{AD}}+\right. \\
& \left.S C \times W_{\mathrm{SC}}+D C \times W_{\mathrm{DC}}\right)
\end{aligned}
$$

It measures reusability with Business commonality, modularity, Adaptability, standard conformance and discoverability.

o Business commonality (BCM): This assesses the degree of functional and non functional commonness of service in the domain.

o Modularity (MD): This measures the degree of independence of service with other services.

o Adaptability (AD): It measures the internal adaptability of the service i.e., how well the service adapts the consumer’s need. o Standard Conformance (SC): This metric measures the degree of service conformance with relevant standards.

o Discoverability: This measures how ease the consumer can correctly discover the service.

- Ashok Goudar [15] proposes metrics for cost savings due to service reusability. The author proposes two metrics for this purpose.

o Business Service Reusability Savings Metrics which quantifies the savings achieved due to the deployment of business process which can be measured by means of Cost per business service and number of consumers.

o Shared Service Reusability Savings metric which quantifies the savings achieved due to the deployment of reusable shared services and can be measured by means of Cost per shared service and number of consumers.

These two metrics are used to measure the percentage cost savings due the service reusability.

- Ingo Pansa et al [16] proposes metric for measuring the reusability of management services. The authors classified service candidates and service interfaces as Management service type, Management capability type, Management area type and Management entity. The reusability of service is measured by means of the amount of classification dimension associated with the service and the total number of applicable classification dimensions.

- Sang Hun Oh et al [17] proposes reusability metrics for cloud services. First the authors are identified the quality attributes of service reusability. Then the metrics were proposed for the defined attributes of understandability, publicity, adaptability and Composability. The proposed metrics for these attributes are : 
o Comprehensibility of Service (CoS): Understandability is measured with comprehensibility of service metric. The service description should be highly understandable. This metric measures the quality of service description. This can be measured as ratio of Number of fields with acceptable readability with total number of fields. Higher the value indicates higher the understandability.

o Awarability of Service (AoS): Publicity is measured with the metric Awarability of service. The service should be publically available for use. Publicity can be measured with the given metric as the ratio of Number of consumers with service discoverability to the total number of consumers. Higher the value indicates the service is easily available to the consumer.

0 Coverage of variability (CoV) and Completeness of variant set (CoA): Adaptability of service can be measured with these two metrics. CoV measures how many places (variation point) service consumers can customize and it can be measured as ratio of Number of variation point supported to the total number of potential variation points. CoA can be measured as ratio of $\mathrm{CoV}$ in each variation points to number of variation points supported in the service. Integrating these two metrics by giving weight value to each gives the value of adaptability. Higher the value indicates the service is easily customized.

o Modularity of Service (MoS) and Interoperability of Service (IoS): Service Composability can be measured with these two metrics which is an important characteristic of service reusability.
Modularity of service can be measured with number of elements with external dependency and total number of elements. Interoperability of service can be measured with number of dependent services with acceptable interaction and total number of dependent services in participating business processes. Integrating and giving weight on these metrics, the Composability of service can be measured. Higher the value indicates that the service can be easily integrated with other services.

The reusability of service is evaluated as integrating and assigning weight values to these four metrics and taking average value. The value lies between 0 and 1 . Higher the value means that the service is more reusable. In this evaluation of service reusability functionality, dependencies are not considered which the important characteristics of service reusability are.

In the above discussed metrics for service reusability, some metrics have taken few design characteristics and some have taken few quality characteristics. Apart from service reusability metrics, service reuse metrics are also available to measure the benefit of service oriented architecture. In a particular time period, the total numbers of services available, number of new consumers, total number of services reused, total effort saved due to reusing of services are taken for measuring the service reuse.

\section{Conclusion}

Service reusability is the prime principle of service oriented architecture. The importance of reusability makes the researchers to show interest over the reusability metrics. Many researchers have been proposed metrics for service reusability. In this paper we have given a study of service reusability. Many reuse metrics have been proposed for different systems like object oriented, component oriented and service oriented systems. But we study and survey the service reusability 
metrics. Out of these some metrics have taken design characteristics of service and some have taken quality characteristics of service. But still there is no complete version. This work can help the researchers for further study and proposal of new metrics and empirical validation of existing metrics.

\section{References}

[1] Arun Sharma, Rajesh Kumar \& P.S. Grover, “A Critical Survey of Reusability Aspects for Component Based Systems", World Academy of Science, Engineering and Technology 33, 2007.

[2] Ann Rockley, with Pamela Kostur and Steve Manning, "Managing Enterprise Content: A Unified Content Strategy" available at http://www.managingenterprisecontent.com/myweb /images/MEC_Chapter_2.pdf

[3] Thomas Erl, "SOA Principles of service Design", Pearson Education 2009.

[4] A. Khoshkbarforoushha, P. Jamshidi, F. Shams, “A Metric for Composite Service Reusability Analysis”, WETSoM’10, May 4, 2010, Cape Town, South Africa, ACM 978-1-60558-976-3/10/05.

[5] Si Won Choi and Soo Dong Kim, “A Quality Model for Evaluating Reusability of Services in SOA", $10^{\text {th }}$ IEEE Conference on E-Commerce Technology and the fifth IEEE conference on Enterprise Computing, E-commerce and E-services.

[6] Julie Street, Hassan Gomaa, "Software Architectural Reuse Issues in Service Oriented Architectures", Proceedings of the $41^{\text {st }}$ Hawaii International Conference on System Sciences-2008, 1530-1605/08, 2008, IEEE.

[7] Marcus A.S.Boxall, Saeed Araban, "Interface Metrics for Reusability Analysis of Components”, Proceedings of the 2004 Australian Software Engineering Conference (ASWEC'04) 15300803/04 2004 IEEE.

[8] P.S. Tan, E.W. Lee, "Standardization of Functionality for Widespread Reuse of Software as Services”, 0-7803-9701-0/06/2006, IEEE.

[9] Arun Sharma, Rajesh Kumar, \& P.S. Grover, "Managing Component Based Systems with Reusable Components", International Journal of Computer Science and Security, Volume 1: Issue (2).

[10] Binghu Shim, Siho Choue, Suntae Kim, Sooyong Park, "A Design Quality Model for Service Oriented Architecture", $200815^{\text {th }}$ Asia Pacific Software Engineering Conference, 1530-1362/08, IEEE.

[11] Renuka Sindhgatta, Bikram Sengupta, and Karthikeyan Ponnalagu, "Measuring the Quality of Service Oriented Design”, ICSOC - Service wave 2009, LNCS 5900, pp 485-499, 2009, Springer Berlin Heidelberg 2009.
[12] Huynh Quyet Thang, Pham Thi Quynh, Tran Quoc Viet, "The Reusability and coupling Metrics for Service Oriented Softwares",

[13] Hironori Washizaki, Hirokazu Yamamoto, and Yoshiaki Fukazawa, "A Metrics Suite for Measuring Reusability of Software Components", Proceedings of the ninth International Software metrics Symposium (Metrics’03) 1530-1435/03, 2003, IEEE.

[14] Si Won Choi and Soo Dong Kim, “A Quality Model for Evaluating Reusability of services in SOA", $10^{\text {th }}$ IEEE Conference on E-Commerce Technology and the Fifth IEEE Conference on Enterprise Computing, E-Commerce and EServices,1530-1354/08,2008,IEEE.

[15] Ashok Goudar, "SOA Measurements and Reporting”, MPHASIS white paper, December, 2008.

[16] Ingo Pansa, Chistoph Leist, Matthias Reichle, Sebastian Abeck, "Designing Reusable Management Services”, Service Computation 2011 : The third International Conference on Advanced Service computing.

[17] Sang hun Oh, Hyun Jung La and Soo Dong Kim, “A reusability evaluation Suite for Cloud services", 978-0-7695-4518-9/11, 2011, IEEE.

\section{AUTHORS}

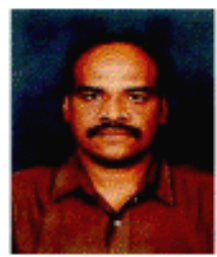

Prof. Thirunavukarasu Karthikeyan received his graduate degree in Mathematics from Madras University in 1982. Post graduate degree in Applied Mathematics from Bharathidasan University in 1984. Received Ph.D. in Computer Science from Bharathiyar University in 2009. Presently he is working as an Associate Professor in Computer Science Department of P.S.G. College of Arts and Science, Coimbatore. His research interests are Image Coding, Medical Image Processing, Data Mining and Software Engineering. He has published many papers in national and international conferences and journals. He has completed many funded projects with excellent comments. He has contributed as a program committee member for a number of international conferences. He is the review board member of various reputed journals. He is board of studies member for various autonomous institutions and universities. He can be contacted by email t.karthikeyan.gasc@gmail.com.

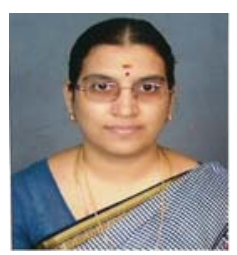

Mrs. J. Geetha received her Post graduate degree in Computer Applications from Madras University in 1996. Received Master of Technology Degree in IT from Allahabad Agricultural Institute Deemed University in 2005. She is now doing her research work in Bharathiyar University in the area of software metrics. Her research areas of interests are Software metrics and Data mining. Presently she is working as an Assistant Professor in the department of Computer Science and Engineering of Sri Manakula Vinayagar Engineering College, Pondicherry. She has published many papers in national and international conferences. She can be contacted by email geethakishorekumar@gmail.com 